\title{
Safety and Efficacy of Intermittent High-Dose Liposomal Amphotericin B Antifungal Prophylaxis in Haemato-Oncology: An Eight-Year Single-Centre Experience and Review of the Literature
}

\author{
Jonathan Youngs ${ }^{1,2,3, *(\mathbb{D})}$, Jen Mae Low ${ }^{4}\left(\mathbb{D}\right.$, Laura Whitney ${ }^{5}$, Clare Logan $1,2,3$, Janice Chase ${ }^{5}$, \\ Ting Yau ${ }^{5}$, Matthias Klammer ${ }^{6}$, Mickey Koh ${ }^{3,6}$ and Tihana Bicanic 1,2,3,* \\ 1 Institute of Infection \& Immunity, St George's University of London, Cranmer Terrace, Tooting, \\ London SW17 0RE, UK \\ 2 Department of Infection, St George's University Hospitals NHS Foundation Trust, Blackshaw Rd, Tooting, \\ London SW17 0QT, UK \\ 3 Clinical Academic Group in Infection and Immunity, St George's University of London, Cranmer Terrace, \\ London SW17 0RE, UK \\ 4 St George's University Hospitals NHS Foundation Trust, Blackshaw Rd, Tooting, London SW17 0QT, UK \\ 5 Pharmacy Department, St George's University Hospitals NHS Foundation Trust, Blackshaw Rd, Tooting, \\ London SW17 0QT, UK \\ 6 Department of Haematology, St George's University Hospitals NHS Foundation Trust, \\ Blackshaw Rd, Tooting, London SW17 0QT, UK \\ * Correspondence: jyoungs@sgul.ac.uk (J.Y.); tbicanic@sgul.ac.uk (T.B.); Tel.: +078-5467-7681 (J.Y.); \\ +020-8725-2911 (T.B.)
}

Received: 21 November 2020; Accepted: 17 December 2020; Published: 21 December 2020

\begin{abstract}
Triazoles remain first-line agents for antifungal prophylaxis in high-risk haemato-oncology patients, but their use is increasingly contraindicated due to drug-drug interactions and additive toxicities with novel treatments. In this retrospective, single-centre, observational study, we present our eight-year experience of antifungal prophylaxis using intermittent high-dose liposomal Amphotericin B (L-AmB). All adults identified through our Antifungal Stewardship Programme as receiving L-AmB prophylaxis at $7.5 \mathrm{mg} / \mathrm{kg}$ once-weekly between February 2012 and January 2020 were included. Adverse reactions, including infusion reactions, electrolyte loss, and nephrotoxicity, were recorded. 'Breakthrough' invasive fungal infection (IFI) occurring within four weeks of L-AmB was classified using European Organization for Research and Treatment of Cancer/Invasive Fungal Infections Cooperative Group and the National Institute of Allergy and Infectious Diseases Mycoses Study Group (EORTC/MSG) criteria. Moreover, 114 courses of intermittent high-dose L-AmB prophylaxis administered to 92 unique patients were analysed. Hypokalaemia was the most common grade 3-4 adverse event, with $26(23 \%)$ courses. Grade 3 nephrotoxicity occurred in $8(7 \%)$ and reversed in all six patients surviving to 90 days. There were two (1.8\%) episodes of breakthrough IFI, one 'probable' and one 'possible'. In this study, the largest evaluation of intermittent high-dose L-AmB prophylaxis conducted to date, toxicity was manageable and reversible and breakthrough IFI was rare. L-AmB prophylaxis represents a viable alternative for patients with a contraindication to triazoles.
\end{abstract}

Keywords: hematopoietic stem-cell transplantation (HSCT); acute leukaemia; graft-versus-host disease (GvHD); antifungal prophylaxis; amphotericin B; triazoles 


\section{Introduction}

Antifungal prophylaxis is recommended for selected patients undergoing cytotoxic chemotherapy and hematopoietic stem-cell transplantation (HSCT) to reduce the risk of invasive fungal infection (IFI) including candidiasis, aspergillosis and mucormycosis [1]. Because of their broad antifungal spectrum, ease of administration, and ability to achieve target serum concentrations, mould-active triazoles (e.g., itraconazole/posaconazole) remain first line agents in high-risk patients such as those with acute myeloid leukaemia/myelodysplastic syndromes (AML/MDS) or undergoing treatment for graft-versus-host disease (GvHD).

Triazole use is, however, often contraindicated due to their hepatic metabolism and toxicity as well as QTc prolongation. Itraconazole and posaconazole are potent inhibitors of cytochrome P450 $3 \mathrm{~A} 4$ and p-glycoprotein, so numerous drug-drug interactions must also be considered [2]. Alongside commonly used drugs a variety of interactions and additive toxicities exist between triazoles and both established and novel treatments for hematological malignancy (Supplementary Table S1) [3-6].

Conventional Amphotericin B deoxycholate (C-AmB) is the most broad-spectrum antifungal agent, with crucial activity against most Aspergillus spp., Candida spp., Mucor spp., and other environmental moulds. Its unfavourable toxicity profile, notably electrolyte abnormalities, nephrotoxicity, and anaemia, has hindered its use as a prophylactic agent $[2,7,8]$. AmBisome ${ }^{\circledR}(\mathrm{L}-\mathrm{AmB})$ is a liposomal formulation of Amphotericin B with a more favorable toxicity profile $[9,10]$. In an RCT of pyrexic neutropenic adults, L-AmB was as effective as C-AmB, but associated with 2-6 times fewer drug-related side effects and severe drug-related side effects were seen in only $1 \%$ vs. $12 \%(p<0.01)$ [10].

To treat IFI, L-AmB is administered daily (dose $3-5 \mathrm{mg} / \mathrm{kg} /$ day) which, given its intravenous route, is impractical for outpatients requiring antifungal prophylaxis. However, when administered at higher doses $(7.5-15 \mathrm{mg} / \mathrm{kg})$, therapeutic levels of L-AmB persist in tissues for over a week without an associated increase in toxicity, supporting intermittent high dosing [2,11-14]. Administering L-AmB at $7.5 \mathrm{mg} / \mathrm{kg}$ (once-weekly) and $15 \mathrm{mg} / \mathrm{kg}$ (once only) to myeloma patients undergoing allograft transplantation resulted in mean serum and buccal concentrations of the drug well in excess of Candida and Aspergillus MICs after seven days [12] and doses up to $7.5-15 \mathrm{mg} / \mathrm{kg} /$ day were well tolerated in a maximally-tolerated dose pharmacokinetic (PK) study, although severe hypokalaemia was more common in patients receiving $>10 \mathrm{mg} / \mathrm{kg} /$ day ( $0 \%$ vs. $35 \%, p=0.006$ ) [15].

Since 1999, 15 studies have reported on the safety and efficacy of different regimens of intravenous L-AmB prophylaxis in haemato-oncology patients: 12 were prospective and $5 \mathrm{RCTs}$, of which only one (2017) trial had $>100$ patients per arm (Table 1) [9,11,12,14,16-27]. All eight cohorts using once-weekly doses $\geq 7.5 \mathrm{mg} / \mathrm{kg}$ have been small ( $<50$ patients). In this single-centre, retrospective observational study, we report our eight-year experience of the safety (toxicity/adverse events) and efficacy (incidence of breakthrough IFI) of L-AmB prophylaxis in high risk haemato-oncology patients encompassing the largest cohort of high-dose, intermittent L-AmB prophylaxis reported to date. 
Table 1. Summary of studies on the use of L-AmB prophylaxis.

\begin{tabular}{|c|c|c|c|c|c|c|c|c|}
\hline $\begin{array}{l}\text { Study Type, Year, } \\
\text { [Reference] }\end{array}$ & $\begin{array}{l}\text { Population (Total } \\
\text { No of Patients) }\end{array}$ & $\begin{array}{l}\text { L-AmB Dosing } \\
\text { Schedule (No. of } \\
\text { Patients) }\end{array}$ & $\begin{array}{l}\text { Nephrotoxicity, No } \\
\text { of Patients (\%) }\end{array}$ & $\begin{array}{l}\text { Hypokalaemia No } \\
\text { of Patients (\%) }\end{array}$ & $\begin{array}{l}\text { Hypomagnasaemia } \\
\text { No of Patients (\%) }\end{array}$ & $\begin{array}{l}\text { Infusion-related } \\
\text { Reaction, No of } \\
\text { Patients (\%) }\end{array}$ & Rate of IFI (Timepoint) & $\begin{array}{l}\text { Mortality } \\
\text { (Timepoint) }\end{array}$ \\
\hline $\begin{array}{c}\text { Prospective, } \\
\text { multi-centre, } \\
\text { double-blind, } \\
\text { randomised } \\
\text { placebo-controlled trial. } \\
1999[9]\end{array}$ & $\begin{array}{c}\text { BMT or intensive } \\
\text { chemotherapy for } \\
\text { haematological } \\
\text { malignancy, Adults } \\
\text { (161) }\end{array}$ & $\begin{array}{l}2 \mathrm{mg} / \mathrm{kg} \text { three times } \\
\text { per week (74) vs. } \\
\text { placebo (87) }\end{array}$ & $\begin{array}{c}\text { Grade } 2-4,9(12 \%) \\
\text { vs. } 6(7 \%)\end{array}$ & $\begin{array}{c}\text { Grade } 4,1(1.4 \%) \\
\text { vs. } 0\end{array}$ & NR & $\begin{array}{l}5(6 \%) \text { leading to } \\
\text { cessation vs. } 1(1 \%)\end{array}$ & $\begin{array}{l}0 \text { proven/probable, } 31 \\
(42 \%) \text { suspected vs. } 3 \\
(3.4 \%) \text { proven/probable, } \\
40(46 \%) \text { suspected with } \\
\text { placebo * (TU). } 15(20 \%) \\
\text { had fungal colonisation } \\
\text { from at least one body } \\
\text { site vs. } 35(40 \%), p<0.01\end{array}$ & $\begin{array}{l}11(15 \%) \text { vs. } 12 \\
(14 \%)(\mathrm{TU}), 1 \text { in } \\
\text { each arm attribute } \\
\text { to IFI ( } 3-182 \text { days } \\
\text { after the end of } \\
\text { the study] }\end{array}$ \\
\hline $\begin{array}{c}\text { Prospective, } \\
\text { single-centre, } \\
\text { non-blinded, } \\
\text { randomised. } 2001 \text { [22] }\end{array}$ & $\begin{array}{l}\text { SCT, intensive } \\
\text { chemotherapy for } \\
\text { haematological } \\
\text { malignancy, MDS, } \\
\text { VSAA, Children } \\
\text { (29) }\end{array}$ & $\begin{array}{l}1 \mathrm{mg} / \mathrm{kg} \text { three times } \\
\text { weekly (16) vs. no } \\
\text { systemic antifungal } \\
\text { prophylaxis (13) }\end{array}$ & 0 & 0 & 0 & $\begin{array}{l}3 / 16(19 \%) \text { leading } \\
\text { to cessation }\end{array}$ & $\begin{array}{c}5 / 16(31 \%) \\
\text { proven/probable vs. 6/13 } \\
(46 \%) \text { (1 year) }\end{array}$ & $\begin{array}{c}0 \text { "by proven IFI" } \\
\text { (1 year) }\end{array}$ \\
\hline $\begin{array}{c}\text { Prospective, } \\
\text { single-centre } \\
\text { randomised. } 2003 \text { [23] }\end{array}$ & $\begin{array}{c}\text { Induction } \\
\text { chemotherapy for } \\
\text { newly diagnosed } \\
\text { AML or high-risk } \\
\text { MDS, Adults (139) }\end{array}$ & $\begin{array}{l}3 \mathrm{mg} / \mathrm{kg} \text { three times } \\
\text { per week (72) vs. } \\
\text { fluconazole plus } \\
\text { itraconazole }(67)\end{array}$ & $\begin{array}{l}\text { Grade } 1,2,14(20 \%) \\
\quad \text { vs. } 4(6 \%)\end{array}$ & NR & NR & $\begin{array}{c}5(7 \%) .10 \\
\text { withdrawn because } \\
\text { of drug-related } \\
\text { toxicities vs. } 5 \text { in } \\
\text { F\&I arm }\end{array}$ & $\begin{array}{c}3(4 \%) \text { proven/probable } \\
\text { vs. } 3(4 \%) \text { (during } \\
\text { prophylaxis) }\end{array}$ & $\begin{array}{c}10 \text { (14\%) vs. } 8(12 \%) \\
1 \text { in each arm } \\
\text { attribute to IFI } \\
\text { (30 days) }\end{array}$ \\
\hline $\begin{array}{c}\text { Prospective, } \\
\text { single-centre, } \\
\text { non-comparative. } \\
2006 \text { [24] }\end{array}$ & $\begin{array}{c}\text { Allo-SCT with } \\
\text { GvHD, Adults (21) }\end{array}$ & $\begin{array}{l}7.5 \mathrm{mg} / \mathrm{kg} \text { once } \\
\text { weekly }(21)^{\ddagger}\end{array}$ & $\begin{array}{l}\text { Grade } 2-4,5(24 \%) \\
\text { leading to cessation }\end{array}$ & NR & NR & $\begin{array}{l}6(29 \%) \text {, leading to } \\
\text { cessation in } 2(10 \%)\end{array}$ & $\begin{array}{c}1 \text { (5\%) proven/probable, } \\
\text { (2 months) }\end{array}$ & $\begin{array}{l}8(38 \%) \text { (median } \\
\text { follow up } 377 \text { days) }\end{array}$ \\
\hline $\begin{array}{c}\text { Prospective, singe } \\
\text { centre, randomized. } \\
2006[25]\end{array}$ & $\begin{array}{l}\text { Intensive } \\
\text { chemotherapy for } \\
\text { hematological } \\
\text { malignancies, } \\
\text { Adults (132) }\end{array}$ & $\begin{array}{l}50 \mathrm{mg} \text { alt days }(75) \\
\text { vs. no systemic } \\
\text { antifungal } \\
\text { prophylaxis (57) }\end{array}$ & 0 grade $3 / 4$ & 0 grade $3 / 4$ & 0 grade $3 / 4$ & $\begin{array}{l}5(7 \%) \text { leading to } \\
\text { cessation in } 3\end{array}$ & $\begin{array}{c}5 / 75(7 \%) \\
\text { proven/probable }(2 \\
\text { Proven IPA, } 3 \\
\text { candidaemia) vs. } 20 / 57 \\
(35 \%) \text { with no } \\
\text { prophylaxis }(p=0.001) \\
\text { (during prophylaxis) }\end{array}$ & $\begin{array}{l}4(5 \%) \text { vs. } 9(16 \%), \\
(p=0.13) \text {, mortality } \\
\text { related to IFI } 2 \text { vs. } 8 \\
(p=0.07) \text { (median } \\
\text { follow up } 17 \text { days) }\end{array}$ \\
\hline $\begin{array}{c}\text { Prospective, } \\
\text { multicentre, } \\
\text { non-comparative. } \\
2007 \text { [14] }\end{array}$ & $\begin{array}{l}\text { Allo-SCT or } \\
\text { chemotherapy for } \\
\text { acute leukaemia } \\
\text { (AL), Adults (29) }\end{array}$ & $\begin{array}{c}10 \mathrm{mg} / \mathrm{kg} \text { once } \\
\text { weekly }(29) \ddagger: 8 \mathrm{SCT} \\
* * \text { and } 21 \mathrm{AL}\end{array}$ & $\begin{array}{l}\text { Grade } 2-4,4(14 \%), \\
\text { lading to cessation } \\
\text { in } 3(10 \%)\end{array}$ & $2(7 \%)$ grade 3 & NR & $\begin{array}{l}12(41 \%) \text { leading to } \\
\text { cessation } 1 \mathrm{AL} \text { and } \\
5 \mathrm{SCT} \text { patients. } 1 \\
\text { case of anaphylaxis. }\end{array}$ & $\begin{array}{c}4(14 \%) \text { proven/ probable } \\
(1 \mathrm{SCT}, 3 \mathrm{AL}) .17(59 \%) \\
\text { switched to empirical } \\
\text { treatment (during } \\
\text { prophylaxis) }\end{array}$ & $\begin{array}{l}4(2 \text { and } 2) \\
\text { (24 weeks) }\end{array}$ \\
\hline $\begin{array}{l}\text { Prospective, single } \\
\text { centre, randomised, } \\
\text { pharmacokinetic. } \\
2009 \text { [12] }\end{array}$ & $\begin{array}{l}\text { Allo and Auto-SCT } \\
\text { for multiple } \\
\text { myeloma, Adults } \\
\text { (16) }\end{array}$ & $\begin{array}{c}15 \mathrm{mg} / \mathrm{kg} \text { once only } \\
\text { (6) vs. } 7.5 \mathrm{mg} / \mathrm{kg} \\
\text { once weekly (4) } \\
1 \mathrm{mg} / \mathrm{kg} \text { daily (6) }\end{array}$ & $\begin{array}{c}\text { Grade } 2-4,3 / 6(50 \%) \\
\text { vs. } 1 / 4(25 \%) \text { vs. } 1 / 6 \\
(17 \%)\end{array}$ & $\begin{array}{c}\text { Grade } 315 \mathrm{mg} / \mathrm{kg}: \\
1 / 6(17 \%) \text { of } \\
\text { patients. Grade } 2 \\
2 / 6(33 \%) \text { vs. } 1 / 4 \\
(25 \%) \text { vs. } 1 / 6(17 \%) .\end{array}$ & $\begin{array}{c}\text { 6/6 (100\%) vs. } 4 / 4 \\
(100 \%) \text { vs. } 5 / 6 \\
(83 \%)\end{array}$ & $\begin{array}{l}1 / 6(17 \%) \text { vs. } 2 / 4 \\
(50 \%), 1 \text { leading to } \\
\text { cessation vs. } 2 / 6 \\
(33 \%), 1 \text { leading to } \\
\text { cessation. }\end{array}$ & NR & NR \\
\hline
\end{tabular}


Table 1. Cont

\begin{tabular}{|c|c|c|c|c|c|c|c|c|}
\hline $\begin{array}{l}\text { Study Type, Year, } \\
\text { [Reference] }\end{array}$ & $\begin{array}{l}\text { Population (Total } \\
\text { No of Patients) }\end{array}$ & $\begin{array}{l}\text { L-AmB Dosing } \\
\text { Schedule (No. of } \\
\text { Patients) }\end{array}$ & $\begin{array}{l}\text { Nephrotoxicity, No } \\
\text { of Patients (\%) }\end{array}$ & $\begin{array}{l}\text { Hypokalaemia No } \\
\text { of Patients (\%) }\end{array}$ & $\begin{array}{l}\text { Hypomagnasaemia } \\
\text { No of Patients }(\%)\end{array}$ & $\begin{array}{l}\text { Infusion-related } \\
\text { Reaction, No of } \\
\text { Patients (\%) }\end{array}$ & Rate of IFI (Timepoint) & $\begin{array}{l}\text { Mortality } \\
\text { (Timepoint) }\end{array}$ \\
\hline $\begin{array}{c}\text { Retrospective, } \\
\text { single-centre, } \\
\text { comparative. 2009 [26] }\end{array}$ & $\begin{array}{c}\text { Induction } \\
\text { chemotherapy for } \\
\text { newly diagnosed } \\
\text { AML or high-risk } \\
\text { MDS, Adults (730) }\end{array}$ & $\begin{array}{c}3 \mathrm{mg} / \mathrm{kg} \text { three times } \\
\text { per week (69) vs. } 9 \\
\mathrm{mg} / \mathrm{kg} \text { once weekly } \\
\text { (27) } \text { also } 6 \text { other } \\
\text { arms AmB Lipid } \\
\text { Complex, Itra-, } \\
\text { flucon plus itra-, } \\
\text { vori-, caspo- }\end{array}$ & NR & NR & NR & $\begin{array}{l}\text { Drug related side } \\
\text { effects } 14 \% \text { vs. } 12 \%\end{array}$ & $3(4 \%)$ vs. $2(7 \%)(\mathrm{TU})$ & NR \\
\hline $\begin{array}{c}\text { Retrospective, } \\
\text { single-centre, } \\
\text { comparative. } 2010 \text { [11] }\end{array}$ & $\begin{array}{c}\text { Allo-SCT with } \\
\text { GvHD, Adults (125) }\end{array}$ & $\begin{array}{l}7.5 \mathrm{mg} / \mathrm{kg} \text { once } \\
\text { weekly (42) })^{\ddagger} \text { vs. } \\
\text { 'Other antifungal } \\
\text { prophylaxis' (83) of } \\
\text { which-59 (72\%) } \\
\text { fluconazole, } 13 \\
(16 \%) \text { other azole }\end{array}$ & $5(12 \%)$, reversible & NR & NR & NR & $\begin{array}{c}4 / 42(10 \%) \\
\text { Proven/Probable plus } 2 \\
(5 \%) \text { Possible. In control } \\
\text { arm 13/83 (16\%) } \\
\text { proven/probable plus } 22 \\
(27 \%) \text { possible (1 year) }\end{array}$ & $\begin{array}{c}14(33 \%) \text { vs. } 35(42 \%) \\
\quad p=0.256) . \\
\text { IFI-related mortality } \\
0 \text { vs. } 12(14 \%)(1 \\
\text { year) }(p=0.005) \\
(1 \text { year })\end{array}$ \\
\hline $\begin{array}{c}\text { Prospective, } \\
\text { single-centre, } \\
\text { comparative. } 2011 \text { [27] }\end{array}$ & $\begin{array}{c}\text { Intensive } \\
\text { chemotherapy for } \\
\text { haematological } \\
\text { malignancy, VSAA, } \\
\text { Children (89) }\end{array}$ & $\begin{array}{l}2.5 \mathrm{mg} / \mathrm{kg} \text { twice } \\
\text { weekly (44) vs. } \\
\text { historical control } \\
\text { group (45) }\end{array}$ & $\begin{array}{l}7(16 \%), \text { with } 1 \\
\text { grade } 3 / 4\end{array}$ & $\begin{array}{c}25 / 184(13.6 \%) \\
\text { grade } 3 / 4,3 / 184 \\
\text { grade } 4\end{array}$ & NR & $\begin{array}{c}\text { 4/184 }(2 \%) \\
\text { prophylaxis } \\
\text { episodes leading to } \\
\text { cessation }\end{array}$ & $\begin{array}{c}0 \text { proven } / \text { probable vs. } 7 \\
(16 \%) \text { in historical control } \\
\text { group }(p=0.01)(2 \\
\text { months })\end{array}$ & $\begin{array}{c}8(18 \%) \text { mortality } \\
\text { (median follow up } \\
29 \text { months) }\end{array}$ \\
\hline $\begin{array}{c}\text { Prospective, single } \\
\text { centre, } \\
\text { non-comparative. } \\
2013[16] \\
\end{array}$ & $\begin{array}{l}\text { Induction } \\
\text { chemotherapy for } \\
\text { AML, Adults, (48) }\end{array}$ & $\begin{array}{l}15 \mathrm{mg} / \mathrm{kg} \text { once only, } \\
\text { followed by second } \\
\text { dose after } 15 \text { days } \\
\text { neutropenia }(48)^{\ddagger}\end{array}$ & $4(8 \%), 0$ grade $3 / 4$ & $6(12.5)$ grade 3 & 0 & $\begin{array}{l}\text { 6/53 (11.3\%) of } \\
\text { infusions cessation } \\
\text { in 1/53(1.9\%) }\end{array}$ & $4(8.3 \%)$ proven (4 weeks) & 13 (27\%) (3 months) \\
\hline $\begin{array}{c}\text { Retrospective, single } \\
\text { centre, } \\
\text { non-comparative. } \\
2014 \text { [17] }\end{array}$ & $\begin{array}{c}\text { ALL, or solid } \\
\text { tumour with } \\
\text { prolonged } \\
\text { neutropenia, } \\
\text { Children (19) }\end{array}$ & $\begin{array}{l}10 \mathrm{mg} / \mathrm{kg} \text { once } \\
\text { weekly }(19)^{\ddagger}\end{array}$ & $\begin{array}{l}1(5 \%) \text { leading to } \\
\text { cessation }\end{array}$ & $7(37 \%)$ grade $1-3$. & $2(10 \%)$ any grade & $\begin{array}{l}5(26 \%) \text { all leading } \\
\text { to cessation }\end{array}$ & $\begin{array}{c}1 \text { possible (5\%) (during } \\
\text { prophylaxis) }\end{array}$ & 1 (5\%) (3 months] \\
\hline $\begin{array}{c}\text { Retrospective, } \\
\text { single-centre, } \\
\text { comparative. } 2014 \text { [18] }\end{array}$ & $\begin{array}{c}\text { Allo-SCT with } \\
\text { GvHD, Adults (101) }\end{array}$ & $\begin{array}{l}3 \mathrm{mg} / \mathrm{kg} \text { once } \\
\text { weekly (16) vs. } \\
\text { echinocandin (12) } \\
\text { vs. triazole (73) }\end{array}$ & 0 discontinuation & 0 discontinuation & NR & 0 discontinuation & $\begin{array}{l}\text { 0/16 proven/probable vs. } \\
\text { 2/12(17\%) vs. } 1 / 73(1 \%) .3 \\
\text { possible }(19 \%) \text { vs. } 0 \text { vs. } \\
3 / 73(4 \%)(p=0.145) \\
\text { (during prophylaxis) }\end{array}$ & $\begin{array}{l}2(12.5 \%) \text { neither } \\
\text { deemed related to } \\
\text { IFI ( } 3 \text { months) }\end{array}$ \\
\hline $\begin{array}{c}\text { Prospective, } \\
\text { single-centre, } \\
\text { non-comparative. } \\
2015 \text { [19] }\end{array}$ & $\begin{array}{l}\text { Liver transplant } \\
\text { recipients with risk } \\
\text { factors for IFI, } \\
\text { Adults (76) }\end{array}$ & $\begin{array}{l}10 \mathrm{mg} / \mathrm{kg} \text { once } \\
\text { weekly }(76)^{\ddagger}\end{array}$ & $3(4 \%)$ grade 3 & 0 (grade $3 / 4$ ) & NR & $\begin{array}{l}6(8 \%) \text { leading to } \\
\text { discontinuation }\end{array}$ & $\begin{array}{c}3(4 \%) \text { proven/probable }(2 \\
\text { invasive candidiasis, } 1 \\
\text { IPA), } 1 \text { possible (during } \\
\text { prophylaxis) }\end{array}$ & $\begin{array}{c}20(26 \%) \text { all-cause, } 0 \\
\text { attributed to IFI } \\
(180 \text { days })\end{array}$ \\
\hline
\end{tabular}


Table 1. Cont.

\begin{tabular}{|c|c|c|c|c|c|c|c|c|}
\hline $\begin{array}{l}\text { Study Type, Year, } \\
\text { [Reference] }\end{array}$ & $\begin{array}{l}\text { Population (Total } \\
\text { No of Patients) }\end{array}$ & $\begin{array}{l}\text { L-AmB Dosing } \\
\text { Schedule (No. of } \\
\text { Patients) }\end{array}$ & $\begin{array}{l}\text { Nephrotoxicity, No } \\
\text { of Patients (\%) }\end{array}$ & $\begin{array}{l}\text { Hypokalaemia No } \\
\text { of Patients (\%) }\end{array}$ & $\begin{array}{l}\text { Hypomagnasaemia } \\
\text { No of Patients (\%) }\end{array}$ & $\begin{array}{l}\text { Infusion-related } \\
\text { Reaction, No of } \\
\text { Patients (\%) }\end{array}$ & Rate of IFI (Timepoint) & $\begin{array}{l}\text { Mortality } \\
\text { (Timepoint) }\end{array}$ \\
\hline $\begin{array}{l}\text { Prospective, } \\
\text { multi-centre, } \\
\text { randomised. } 2015 \text { [20] }\end{array}$ & $\begin{array}{l}\text { Induction } \\
\text { chemotherapy for } \\
\text { haematological } \\
\text { malignancy, Adults } \\
\text { (52) }\end{array}$ & $\begin{array}{c}1 \mathrm{mg} / \mathrm{kg} \text { daily }(16), 3 \\
\mathrm{mg} / \mathrm{kg} \text { twice weekly } \\
\text { (3), 3 mg/kg three } \\
\text { times weekly (18) or } \\
10 \mathrm{mg} / \mathrm{kg} \text { once } \\
{\text { weekly }(15)^{\ddagger}}^{\ddagger}\end{array}$ & NR & NR & NR & $\begin{array}{c}\mathrm{AE} \text { in } 6 / 16(37.5 \%), \\
2 / 3(66.7 \%), 10 / 18 \\
(55.6 \%) \text { and } 9 / 15 \\
(60 \%) \text { respectively. } \\
\text { Serious AE in } 1 / 16 \\
(6.3 \%), 0 / 3(0 \%), \\
2 / 18(11.1 \%) \text { and } \\
2 / 15(13.3 \%)\end{array}$ & $\begin{array}{c}\text { Proven/probable in } 1 / 16 \\
(6.3 \%), 0 / 3(0 \%), 2 / 18 \\
(11.1 \%) \text { and } 1 / 15(6.7 \%) \\
\text { respectively }(p=0.89) \\
\text { (TU) }\end{array}$ & $\begin{array}{c}5 / 16(31.3 \%), 1 / 3 \\
(33.3 \%), 1 / 18(5.6 \%) \\
\text { and } 4 / 15(26.7 \%), \\
\text { respectively }(p= \\
0.25) .2 \text { IFD-related } \\
\text { deaths in the } 1 \\
\text { mg/kg daily arm } \\
\text { but no other } \\
\text { IFD-related deaths. } \\
\text { (52 days) }\end{array}$ \\
\hline $\begin{array}{l}\text { Prospective, } \\
\text { multi-centre, } \\
\text { double-blind, } \\
\text { randomised } \\
\text { placebo-controlled trial. } \\
2017 \text { [21] }\end{array}$ & $\begin{array}{l}\text { Induction } \\
\text { chemotherapy for } \\
\text { ALL, Adults. (355) }\end{array}$ & $\begin{array}{c}5 \mathrm{mg} / \mathrm{kg} \text { twice } \\
\text { weekly (237) vs. } \\
\text { placebo (118) }\end{array}$ & $\begin{array}{c}22 / 237(9 \%) \\
\text { creatinine increased } \\
\text { but } 0 \text { grade } 3 / 4 \text { vs. } 0 \\
\text { with placebo }\end{array}$ & $\begin{array}{c}\text { 83/237 (35\%) of } \\
\text { which } 13(5 \%) \\
\text { grade } 3 / 4 \text { vs. } \\
21 / 118(18 \%) \text { of } \\
\text { which 3 }(3 \%) \text { grade } \\
\text { 3/4 with placebo }\end{array}$ & NR & $\begin{array}{l}\text { AE leading to study } \\
\text { drug } \\
\text { discontinuation } \\
63 / 237(27 \%) \text { vs. } \\
26 / 118(22 \%)\end{array}$ & $\begin{array}{c}18 / 228(8 \%) \\
\text { proven/probable vs. } \\
13 / 1111(12 \%) \text { in the } \\
\text { placebo group }(p=0.24) . \\
11 / 228(5 \%) \text { possible vs. } \\
6 / 111(5 \%) \text { in the placebo } \\
\text { group }(p=0.82) \text { (30 days) }\end{array}$ & $\begin{array}{c}17 / 237(7 \%) \text { vs. } \\
8 / 118(7 \%) \text { for } \\
\text { placebo }(p=1.00) \\
\quad(30 \text { days })\end{array}$ \\
\hline
\end{tabular}

AE, Adverse event; ALL, acute lymphoblastic leukaemia; Allo, allogenic; BMT, bone marrow transplant; IFI, invasive fungal infection; IPA, invasive pulmonary aspergillosis; L-AmB; Liposomal Amphotericin B; MDS, Myelodysplastic Syndrome; NR, not reported; SCT, stem cell transplant; TU, timepoint unclear; VSAA, Very Severe Aplastic Anaemia. * Proven fungal infection was defined as the microbiological identification of a fungal pathogen associated with clinical or radiological evidence of a disease process likely to be caused by this pathogen. Suspected systemic fungal infection was defined as the presence of fever $\left(37.5^{\circ} \mathrm{C}\right)$ refractory to at least $96 \mathrm{~h}$ of therapy with broad-spectrum antibacterial agents or the presence of symptoms or signs consistent with invasive fungal infection, excluding superficial mucocutaneous infection, while on antibacterial therapy ${ }^{* *}$ Enrolment discontinued in the SCT group, as recommended by the independent data review committee, in accordance with the study protocol ${ }^{\ddagger}$ Intermittent weekly high-dose L-AmB regimens. 


\section{Materials and Methods}

St George's hospital is a 1300-bed London tertiary referral centre treating patients with haematological malignancy. It performs autologous and allogeneic HSCT; 29/year in 2009 rising to an average of 50/year currently. Since October 2010, an Antifungal Stewardship (AFS) programme has operated, incorporating weekly review of antifungal prescriptions for all adult inpatients and Haematology Day Care Unit attendees.

Over the study period, our local antifungal policy recommended itraconazole and posaconazole as 1st and 2nd line prophylactic agents for patients at high risk of IFI [1], who are routinely cared for in positive-pressure HEPA-filtered single rooms. Where triazoles were contraindicated, not tolerated, or deemed unsuitable, L-AmB was used as a 3rd line agent. Before February 2011, L-AmB was administered at $1 \mathrm{mg} / \mathrm{kg} /$ day. Between February 2011-February 2012, the policy changed to $3 \mathrm{mg} / \mathrm{kg}$ $\times$ 3/week. In February 2012, following a positive report from another London institution [28] and literature review of intermittent high-dose L-AmB prophylaxis, a regimen of $7.5 \mathrm{mg} / \mathrm{kg}$ once-weekly was instituted and continues to the present day. Where antifungal prophylaxis relates to intensive chemotherapy (or a novel agent), it is usually commenced at the time of, or prior to, the first dose.

Our local policy is that after a $1 \mathrm{mg}$ test dose, L-AmB is infused over $2 \mathrm{~h}$ without routine pre-medication in the first instance. In the event of a reaction, the infusion rate is slowed and medications (e.g., chloramphenamine, paracetamol, and hydrocortisone) are administered at the discretion of the treating physician for both the current and future infusions.

At-risk patients (e.g., all those receiving antifungal prophylaxis) undergo investigation for IFI if there is persistent fever $(>72 \mathrm{~h})$ despite broad-spectrum antibiotics accompanied by suggestive symptoms or signs. This investigation comprises blood cultures, a CT chest (plus CT sinus/head/abdomen as indicated), serum galactomannan and serum beta-D-glucan. A bronchoalveolar lavage (BAL, with prolonged fungal culture, galactomananan and Pneumocystis pneumonia polymerase chain reaction testing) is pursued in response to $C T$ chest findings. Whilst these investigations are ongoing, prophylaxis is switched to empirical pre-emptive treatment with L-AmB ( $3 \mathrm{mg} / \mathrm{kg} / \mathrm{day})$ if the patient is unwell. Voriconazole is initiated if investigations suggest invasive aspergillosis. Asymptomatic patients are not routinely screened for IFI.

As part of ongoing clinical service evaluation, records of AFS reviews, including patient demographics, antifungal drug/indication and recommendations made, were prospectively entered into secure Microsoft Excel (v2003) and subsequently REDCap databases. We conducted a retrospective evaluation of L-AmB prophylaxis in haemato-oncology patients, extracting data on any patient receiving 'L-AmB' for the indication 'prophylaxis' between October 2010-January 2020 but only patients receiving intermittent high-dose $\mathrm{L}-\mathrm{AmB}(7.5 \mathrm{mg} / \mathrm{kg}$ once weekly) were included in the main analysis. Patients without an underlying haematological condition, primarily under the care of another medical institution or for whom the indication for L-AmB was treatment rather than prophylaxis, were also excluded.

Information extracted included: underlying haematological condition (including HSCT); rationale for antifungal prophylaxis; reason triazole contraindicated; L-AmB dosing/duration and reason for ceasing L-AmB. Missing data were retrieved from paper and electronic medical records and pharmacy, laboratory, and microbiology databases.

Adverse reactions to L-AmB were recorded including nephrotoxicity and hypokalaemia or hypomagnesaemia related to L-AmB administration. Toxicity was graded as per the Common Terminology Criteria for Adverse Events [29].

Episodes of 'breakthrough' IFI were classified as 'proven', 'probable' or 'possible' based on EORTC/MSG criteria [30]. In line with a consensus statement [31] we took the date of breakthrough IFI as the first radiological/clinical sign/mycological finding within the period of antifungal prophylaxis. This in turn depends on antifungal drug half-life and dosing schedule: we defined L-AmB prophylaxis course length as extending until the next dose would be due (i.e., one week with once-weekly 
prophylaxis) but included any IFI within 4 weeks of the last dose of L-AmB as a breakthrough, based on the above PK data.

Data were analysed using Microsoft Excel v2019 and GraphPad Prism v8.4.2 (GraphPad software, LLC, San Diego, CA, USA), using Fischer's exact test for categorical variables and Mann-Whitney U test for continuous variables.

After utilising the NHS Health Research Authority Decision Tools [32], no formal ethics approval was considered necessary as persons accessing patient-identifiable data were members of the direct care team doing so as part of a clinical service evaluation.

\section{Results}

During the nine-year period from October 2010 to January 2020, 147 L-AmB prophylaxis courses were identified. Thirty-three were excluded: low-dose courses (daily $1 \mathrm{mg} / \mathrm{kg} / \mathrm{d}$ or thrice weekly $3 \mathrm{mg} / \mathrm{kg}, n=24)$, treatment not prophylaxis $(n=5)$; patient primarily under another institution $(n=2)$; no underlying hematological condition $(n=2)$. The remaining 114 courses of intermittent high-dose $(7.5 \mathrm{mg} / \mathrm{kg}$ weekly) L-AmB prophylaxis administered to 92 unique patients were analysed (all administered after February 2012). These courses amounted to 520 L-AmB infusions and 3640 patient-days of antifungal prophylaxis.

Baseline characteristics and rationale for L-AmB prophylaxis are shown in Table 2. Median (IQR) patient age was 42 (29-57) years and 57\% were male. The median course length was 24 (7-147) days deriving from 3 (1-21) infusions. The most common indication for antifungal prophylaxis was intensive chemotherapy/novel agent for induction/relapse (77\%), the majority $(78 \%)$ of whom had acute leukaemia. Fourteen patients $(12 \%)$ received antifungal prophylaxis in relation to HSCT and ten $(9 \%)$ due to GvHD. The most frequent contra-indications to a triazole were to avoid a drug-drug interaction (51\%) or abnormal liver-function tests (39\%). Drug-drug interactions were usually related to vincristine or myelotarg ( $88 \%)$.

Table 2. Baseline characteristics and rationale for intermittent high-dose L-AmB prophylaxis $(n=114)$.

\begin{tabular}{|c|c|c|}
\hline Characteristic & \multicolumn{2}{|c|}{ Frequency } \\
\hline Age, Median (IQR) & \multicolumn{2}{|c|}{$42(29-57)$} \\
\hline Male, $n(\%)$ & 65 & $57 \%$ \\
\hline \multicolumn{3}{|c|}{ Rationale for antifungal prophylaxis } \\
\hline $\begin{array}{l}\text { Intensive chemotherapy/novel agent } \\
\text { (induction/relapse) }^{a}\end{array}$ & 88 & $77 \%$ \\
\hline HSCT (All) & 14 & $12 \%$ \\
\hline Autograft (pre-engraftment) $b$ & 7 & $6 \%$ \\
\hline Allograft $(<100 \text { days post- })^{c}$ & 7 & $6 \%$ \\
\hline Immunosuppression for GvHD ${ }^{d}$ & 10 & $9 \%$ \\
\hline Prolonged neutropenia (disease related) ${ }^{e}$ & 2 & $2 \%$ \\
\hline \multicolumn{3}{|c|}{ Rationale for L-AmB (over triazole) } \\
\hline Triazole drug-drug interaction & 58 & $51 \%$ \\
\hline Vincristine & 45 & $39 \%$ \\
\hline Myelotarg & 6 & $5 \%$ \\
\hline Other $^{\mathrm{f}}$ & 7 & $6 \%$ \\
\hline Abnormal liver function tests & 44 & $39 \%$ \\
\hline Unable to tolerate $\mathrm{PO} /$ absorption concerns & 6 & $5 \%$ \\
\hline Cardiomyopathy/risk of QTc prolongation & 3 & $3 \%$ \\
\hline Previous side effects/intolerance to triazole & 3 & $3 \%$ \\
\hline
\end{tabular}


Table 2. Cont.

\begin{tabular}{lcc}
\hline \multicolumn{1}{c}{ Characteristic } & Frequency \\
\hline \multicolumn{1}{c}{ Reason L-AmB prophylaxis discontinued } \\
\hline Triazole no longer contraindicated & 69 & $61 \%$ \\
Antifungal prophylaxis no longer indicated & 16 & $14 \%$ \\
Death/palliation & 7 & $6 \%$ \\
Infusion reaction & 7 & $6 \%$ \\
Nephrotoxicity & 5 & $4 \%$ \\
Confirmed/suspected IFI & 3 & $3 \%$ \\
Acute allergic reaction & 5 & $4 \%$ \\
Other g & 2 & $2 \%$ \\
\hline
\end{tabular}

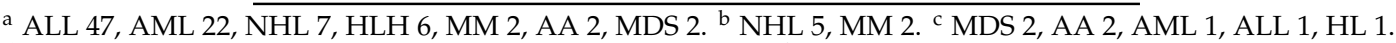
${ }^{\mathrm{d}}$ ALL 4, AML 2, AA 1, MDS 1, MF 1, HL 1. ${ }^{\mathrm{e}}$ AML 1, AA 1. ${ }^{\mathrm{f}}$ Other triazole drug-drug interactions included: Ritonavir $(n=1)$, Thiotepa $(n=2)$, Rifater/Rifanah $(n=2)$, Ethambutol $n=1)$, Imatinib $(n=1)$, Colchicine $(n=1)$. $\mathrm{g}$ Other reason L-AmB discontinued: Transfer of care $(n=1)$, Noted to have reduced eGFR at baseline $(n=1)$. AA, Aplastic anaemia. ALL, Acute lymphocytic leukaemia. AML, Acute myeloid leukaemia. GvHD, Graft vs. Host Disease. HL, Hodgkin lymphoma. HLH, Hemophagocytic lymphohistiocytosis. HSCT, Hematopoietic stem cell transplantation. IFI, Invasive fungal infection. L-AmB, Liposomal Amphotericin B. MDS, Myelodysplastic syndrome. MM, Multiple myeloma. MF, Myelofibrosis. NHL, Non-Hodgkin lymphoma. PO, Per oral.

Of the 88 patients receiving antifungal prophylaxis in relation to intensive chemotherapy/novel agent, $79(90 \%)$ were severely neutropenic $\left(<0.5 \times 10^{9} / \mathrm{L}\right)$ at some point during their L-AmB course. Severe neutropenia lasted over 10 days in $56(71 \%)$ of these courses and occurred for a median of three days (IQR, 0-7) after L-AmB commenced.

Adverse events (AEs) and incidence of breakthrough IFI are summarised in Table 3. Nephrotoxicity and electrolyte loss were the most frequent AEs. The most common grade 3-4 AE was hypokalaemia; occurring in $26(23 \%)$ of L-AmB courses. Hypomagnesemia was common $(66 \%)$ but only one instance was grade 3-4. Grade 3 nephrotoxicity was associated with eight (7\%) L-AmB courses, with no instances of grade 4 nephrotoxicity. Of these eight patients, seven had acute leukaemia and the rationale for prophylaxis was either allograft $(n=3)$, intensive chemotherapy $(n=4)$ or $\operatorname{GvHD}(n=1)$ so all were likely to be receiving concomitant nephrotoxic medications. Excluding two patients who died $<30$ days, creatinine returned to baseline in the remaining six by 90 days ( 3 within30 days) [33]. All infusion reactions were mild but 3 of 5 acute allergic reactions were grade $3-4$. L-AmB prophylaxis was discontinued due to toxicity in $17(15 \%)$ of courses; infusion reaction $(n=7)$, acute allergic reaction $(n=5)$ and nephrotoxicity $(n=5)$ (Table 2).

Table 3. Adverse events, breakthrough IFI and mortality $(n=114)$.

\begin{tabular}{lcc}
\hline L-AmB Prophylaxis Duration & \\
\hline Course length, median days (range) & $24(7-147)$ \\
Number of infusions, median (range) & $3(1-21)$ \\
\hline \multicolumn{1}{c}{ Adverse events ${ }^{\text {a }}$} & \\
\hline Acute allergic reaction & 5 & $4 \%$ \\
Grade 3 & 2 & $2 \%$ \\
Grade 4 & 1 & $1 \%$ \\
Infusion reaction & 7 & $6 \%$ \\
Nephrotoxicity & 65 & $57 \%$ \\
Grade 3 & 8 & $7 \%$ \\
Hypokalaemia & 75 & $66 \%$ \\
Grade 3 & 22 & $19 \%$ \\
Grade 4 & 4 & $4 \%$ \\
Hypomagnesaemia & 75 & $66 \%$ \\
Grade 3 & 1 & $1 \%$ \\
Breakthrough IFI & 2 & $2 \%$ \\
Possible & 1 & $1 \%$ \\
Proven/Probable & 1 & $1 \%$ \\
90 day mortality & 10 & $9 \%$ \\
With IFI ( $n=2)$ & 2 & $100 \%$ \\
Without IFI $(n=112)$ & 8 & $7 \%$ \\
\hline
\end{tabular}

\footnotetext{
a Reported as any grade, followed by grade 3-4 reported separately if present. i.e., For 'infusion reaction' all reactions were grade 1, 2. IFI, Invasive fungal infection. L-AmB, Liposomal Amphotericin B.
} 
In 114 courses of high dose intermittent L-AmB prophylaxis there were just $2(1.8 \%)$ episodes of breakthrough IFI. This incidence remained low $(2.4 \%)$ even if only patients conventionally considered high-risk are considered, e.g., intense chemotherapy for AML/MDS $(n=24)$, treatment for GvHD $(n=10)$, and $<100$ days post allograft $(n=7)$. One patient undergoing intensive chemotherapy for HLH developed 'probable' IFI 7 days into L-AmB prophylaxis [30]. Chest imaging revealed new nodular perihilar air space opacities and Aspergillus fumigatus was isolated twice from sputum culture. The second patient had a background of sibling allograft for ALL and developed 'possible' IFI two weeks into L-AmB prophylaxis for immunosuppression related to GvHD. CT chest revealed bilateral peribronchovascular consolidation with areas of discrete nodules and surrounding ground-glass change supportive of fungal infection. Sputum culture and serum galactomannan were negative, but serum beta-D-glucan was raised at $469 \mathrm{pg} / \mathrm{mL}$. Both patients died within 90-days whereas the 90-day mortality for those without IFI was 8/112 (7\%) (Table 3).

A sensitivity analysis was performed comparing intermittent high-dose L-AmB to the historic cohort of 24 patients that received low-dose AmB prophylaxis between 2010-11. There were no significant differences in nephrotoxicity (any grade, $57 \%$ vs. $42 \%, p=0.18$ ) (grade $3-4,7 \%$ vs. $8 \%$, $p=0.69$ ), hypokalaemia (any grade, $66 \%$ vs. $79 \%, p=0.24$ ) (grade $3-4,23 \%$ vs. $37.5 \%, p=0.1$ ), hypomagnesaemia $(66 \%$ vs. $88 \%, p=0.05)$ or proven/probable IFI ( $1 \%$ vs. $4 \%, p=0.3)$.

\section{Discussion}

Whilst novel targeted therapies represent an exciting development in the treatment of haematological malignancy they present new challenges for antifungal prophylaxis [5]. Drugs such as inotuzumab ozogamicin, ibrutinib, FLT3 inhibitors and venetoclax all have significant interactions/additive toxicities with triazoles and some, such as ibrutinib, are associated with a high incidence of IFI $[34,35]$. Safe, effective oral alternatives to azoles are urgently needed, with several promising novel agents in clinical development [36-40]. In the meantime, intravenous agents remain the most attractive option (although nebulised L-AmB may be an alternative [41]). Whilst echinocandins are an option, they have a narrower spectrum than L-AmB, require daily intravenous dosing, and are fungistatic (rather than -cidal) against Aspergillus spp. [2]. Our eight-year experience of prophylactic L-AmB in high risk haemato-oncology patients includes the largest series of intermittent high-dose courses to date, given for a median of 3-4 weeks. We found that these prophylactic regimens were well tolerated with reversible toxicities and associated with a low $(1.8 \%)$ rate of breakthrough IFI.

The pharmacokinetics of L-AmB support intermittent high-dose administration for several reasons. Firstly, the efficacy of L-AmB is concentration-dependent so achievement of a high maximum drug concentration in plasma (Cmax) compared to the fungal MIC - the Cmax/MIC ratio, is desirable [2,11,12]. Secondly, L-AmB (more so than C-AmB) accumulates in the reticulo-endothelial system and other tissues for several weeks after administration, in a dose-dependent manner [13]. Lastly, L-AmB exhibits non-linear pharmacokinetics meaning that it is preferentially cleared at higher doses [14].

These characteristics mean that when administered at higher doses, therapeutic levels of L-AmB persist in tissues without an associated increase in toxicity. In one study, levels of L-AmB above the MIC of most fungi were maintained for one week in mice lungs and six weeks in kidney/spleen after administration of $15 \mathrm{mg} / \mathrm{kg} \times 3 /$ week for 2-5 weeks. Drug levels in kidney/spleen 3-6 weeks post treatment were similar whether a daily $(2.4 \mathrm{mg} / \mathrm{kg} \times 5 /$ week $)$ or intermittent $(15 \mathrm{mg} / \mathrm{kg}$ once-weekly) dose was used [42]. In another study, a single high-dose of L-AmB protected immunosuppressed mice against challenge with Histoplasma spp. seven days later and survival increased with higher dosing: $20 \%(5 \mathrm{mg} / \mathrm{kg})$ to $60 \%(10 \mathrm{mg} / \mathrm{kg})$ and $80 \%(20 \mathrm{mg} / \mathrm{kg})[43]$.

The largest clinical trial of standard-dose L-AmB prophylaxis to date has been the AmbiGuard study $(n=355)$, a multi-centre randomized placebo-controlled trial of L-AmB prophylaxis $(5 \mathrm{mg} / \mathrm{kg}$ twice-weekly) in induction chemotherapy for ALL (Table 1) [21]. There were more serious AEs considered related to the study drug with L-AmB compared to placebo ( $8 \%$ vs. $2 \%, p=0.02)$ as well as more hypokalaemia (any grade $-35 \%$ vs. $18 \%, p<0.001$ ) and creatinine elevation (any grade $-9 \%$ vs. 
$0 \%, p<0.001)$. Interestingly, however, patients receiving placebo were as likely to have their study drug stopped due to an $\mathrm{AE}$ as those receiving $\mathrm{L}-\mathrm{AmB}(22 \%$ vs. $27 \%, p=0.37)$, suggesting that some of the toxicity observed with L-AmB may be due to concurrent chemotherapy/HSCT conditioning.

In our study, as in others, the most common L-AmB toxicities were renal. Electrolyte loss and nephrotoxicity occurred in $66 \%$ and $57 \%$ of patients respectively. Grade 3 nephrotoxicity occurred in $7 \%$, possibly exacerbated by concomitant use of other nephrotoxic agents, and was reversible in all survivors by 90 days. Grade 3-4 hypokalaemia occurred in $23 \%$ of patients, a figure between that of the placebo and L-AmB arms of AmbiGuard (18\% and 35\%, $p<0.001)$ (Table 3). Careful monitoring and pre-emptive potassium and magnesium replacement can mitigate the risk of this predictable AmB-related AE [44]. In patients with deranged liver function tests the risk of reversible renal toxicity with L-AmB must be weighed against the risk of drug induced liver injury with triazoles. Our policy is to continue with triazoles in patients with cholestatic liver enzyme derangement (alkaline phosphatase and gamma glutamyltransferase) and modest elevations in transaminases (alanine transaminase $<150 \mathrm{IU} / \mathrm{L}$ ), with exclusion of other causes and close laboratory monitoring for worsening transaminitis

In a sensitivity analysis we found no suggestion of increased AEs associated with intermittent high-dose L-AmB compared to the 24 patients that received lower doses, a finding echoed in other studies. In a retrospective analysis of adults with newly diagnosed AML/high-risk MDS, drug-related side effects were similar between patients receiving L-AmB prophylaxis $3 \mathrm{mg} / \mathrm{kg} \times 3 /$ week and $9 \mathrm{mg} / \mathrm{kg}$ once-weekly (14\% vs. 12\%) [26]. In one study that employed L-AmB at $15 \mathrm{mg} / \mathrm{kg}$, there were mild infusion-related reactions in only six out of 53 (11\%) of infusions and only one led to cessation [16]. The authors postulated their long infusion time of $6 \mathrm{~h}$ reduced AEs (our local policy is to administer over $2 \mathrm{~h}$ ).

Our $15 \%$ rate of discontinuation due to toxicity is lower than that seen in a prospective study using the same L-AmB dose (33.3\%) [24] and AmbiGuard (27\%) [21]. This may reflect the patient popuations involved or protocol-driven discontinuationin a clinical trial. In the PROPHYSOME study [14] the trial group suspended enrolment into the SCT arm after AEs led to four of eight patients discontinuing L-AmB (10 mg/kg once-weekly) after the first dose [14]. All patients received at least one concomitant nephrotoxic medication and all eight SCT patients received cyclosporin. Interestingly, $\mathrm{L}-\mathrm{AmB}$ was better tolerated in patients receiving chemotherapy for acute leukaemia, which represents most $(76 \%)$ of our intermittent high-dose cohort. We too found suggestion that L-AmB toxicity prompting discontinuation may be more common with HSCT than with intensive chemotherapy, although the difference was not statistically significant, with four out of $14(29 \%)$ vs. 12 out of 87 $(14 \%)(p=0.2)$. HSCT patients are frequently on concomitant nephrotoxic agents such as ciclosporin, acyclovir and aminoglycosides.

In our cohort, only two (1.8\%) of $114 \mathrm{~L}-\mathrm{AmB}$ courses were associated with breakthrough IFI, of which only one was proven/probable. This rate remained low $(2.4 \%)$ when including only patients conventionally considered high-risk for IFI. Studies on L-AmB prophylaxis report IFI occurring over varying time periods ranging between only during prophylaxis to up to a year after the last dose (Table 1). This may partly explain why our incidence of proven/probable IFI is lower than the $5-10 \%$ reported $[11,21,24]$ elsewhere.

Prospective comparative data on the efficacy of intermittent high-dose L-AmB prophylaxis are scarce. In a prospective study by El-Cheikh et al., (2007) 21 adults receiving high-dose steroids for GvHD post-allograft transplantation were administered L-AmB prophylaxis $7.5 \mathrm{mg} / \mathrm{kg}$ once-weekly. Only one (5\%) instance of proven/probable IFI was observed, two months after discontinuation of L-AmB prophylaxis [24]. In a subsequent retrospective study, the rate of proven/probable IFD was four out of $42(10 \%)$ in the L-AmB group vs. 13 out of $83(16 \%)$ in a comparator group of patients that received 'other antifungal prophylaxis' (a triazole in $87 \%$ ) [11].

In terms of regular standard-dose L-AmB prophylaxis, in AmbiGuard (5 mg/kg twice-weekly) [21], there were fewer episodes of proven/probable IFI with L-AmB compared to placebo, but the difference 
was not statistically significant: 18 in $228(7.9 \%)$ vs. 13 in $111(11.7 \%)(p=0.24)$, possibly due to study underpowering. In a single-centre RCT by Mattiuzzi et al. [23] the incidence of IFI was the same (4\%) in both L-AmB ( $3 \mathrm{mg} / \mathrm{kg} \times 3 /$ week $)$ and fluconazole+itraconazole arms. Finally, in a multi-centre RCT from 1999 [9], there was no 'proven' IFI (broadly similar to EORTC/MSG's 'probable' definition [30]) in the L-AmB arm $(2 \mathrm{mg} / \mathrm{kg} \times 3 /$ week $)$ vs. three $(3.4 \%)$ with placebo. This surprisingly low rate of IFI with placebo may stem from the lack of diagnostics, including fungal biomarkers such as galactomannan, available at the time.

Although clinical guidelines exist for diagnostic sampling in high-risk haematology patients, IFI cases may have been missed in this retrospective evaluation. Under a prospective study protocol more instances of clinical deterioration may have been classified as 'possible' IFI, and with increased utilisation of bronchoscopy more diagnoses of 'probable' IFI might have been made. Inferring a direct link between L-AmB and rates of breakthrough IFI, in particular comparisons between doses and regimens, are limited by historic trends and the absence of a comparator group. Similarly, it is difficult to ascertain whether toxicity observed was directly attributable to L-AmB given the intensive chemotherapy/HSCT conditioning that our patients were also receiving. Moreover, some AEs not recorded in patient notes (e.g., mild infusion reactions) may have been missed.

\section{Conclusions}

To conclude, with an ever-expanding list of triazole drug-drug interactions and additive toxicities to consider, in our large single-centre experience, intermittent high-dose L-AmB prophylaxis represents a safe and effective alternative in high risk haemato-oncology patients. To mitigate the inevitable but reversible common renal toxicities of $\mathrm{L}-\mathrm{AmB}$, we recommend stringent monitoring and pre-emptive hydration and electrolyte replacement, especially in the context of HSCT patients on concomitant nephrotoxic agents. As the list of novel immunosuppressive agents associated with IFI risk expands, prospective, adequately powered non-inferiority trials comparing L-AmB to triazoles and novel oral antifungal prophylactic agents are needed to address the ever-present threat that IFIs pose to advances in the treatment of haematological malignancy.

Supplementary Materials: The following are available online at http://www.mdpi.com/2309-608X/6/4/385/s1, Supplementary Table S1: Mould-activet triazole drug-drug interactions and additive toxicities commonly encountered in haemato-oncology.

Author Contributions: J.Y. and T.B. conceived the idea for this service evaluation and identified key outcome measures and analyses; J.Y. performed data extraction, analysis and drafted the manuscript; J.M.L. assisted with data extraction and analysis; L.W. and T.B. developed the AFS database; L.W. and C.L. undertook primary data collection and data entry; L.W., J.C., and T.Y. assisted with Supplementary Table S1 and pharmacology aspects; M.K. (Mickey Koh) and M.K. (Matthias Klammer) assisted with breakthrough IFI classification, results reporting and contextualisation of key findings within the changing haematology treatment landscape. All authors have read and agreed to the published version of the manuscript.

Funding: J.Y. and C.L. are funded through Gilead UK and Ireland Fellowships awarded to T.B. for separate ICU research in AFS and aspergillosis in influenza [45]. Gilead Sciences had no role in the design, conduct, analysis or write-up of this work.

Conflicts of Interest: T.B. has received speaking fees from Pfizer/Gilead Sciences and Advisory Board fees from Basilea/Gilead Sciences. L.W. has received conference sponsorship and speaker fees from Gilead Sciences, Astellas, Pfizer and Eumedica. T.Y. has received conference sponsorship from Gilead Sciences. M.K. (Mickey Koh) and M.K. (Matthias Klammer) has received conference sponsorship and speaker fees from Gilead Sciences. All other authors declare no conflicts of interest.

\section{References}

1. Taplitz, R.A.; Kennedy, E.B.; Bow, E.J.; Crews, J.; Gleason, C.; Langston, A.A.; Flowers, C.R.; Hawley, D.K.; Nastoupil, L.J.; Rolston, K.V.; et al. Antimicrobial Prophylaxis for Adult Patients with Cancer-Related Immunosuppression: ASCO and IDSA Clinical Practice Guideline Update. J. Clin. Oncol. 2018, 36, 3043-3054. [CrossRef] 
2. Bellmann, R.; Smuszkiewicz, P. Pharmacokinetics of Antifungal Drugs: Practical Implications for Optimized Treatment of Patients. Infection 2017, 45, 737-779. [CrossRef]

3. Stockley, I.; Baxter, K.; Preston, C. Stockley's Drug Interactions. Available online: www.new.medicinescomplete. com (accessed on 26 July 2020).

4. Lexicomp ${ }^{\circledR}$. Drug Interactions. Available online: http://online.lexi.com (accessed on 26 July 2020).

5. Lindsay, J.; Teh, B.W.; Micklethwaite, K.; Slavin, M. Azole Antifungals and New Targeted Therapies for Hematological Malignancy. Curr. Opin. Infect. Dis. 2019, 32, 538-545. [CrossRef]

6. National Aspergillosis Centre Fungal Infection Trust. Antifungal Drug Interactions Database. Available online: https://antifungalinteractions.org (accessed on 20 November 2020).

7. Bodey, G.P.; Anaissie, E.J.; Elting, L.S.; Estey, E.; O’Brien, S.; Kantarjian, H. Antifungal Prophylaxis during Remission Induction Therapy for Acute Leukemia Fluconazole versus Intravenous Amphotericin B. Cancer 1994, 73, 2099-2106. [CrossRef]

8. Wolff, S.N.; Fay, J.; Stevens, D.; Herzig, R.H.; Pohlman, B.; Bolwell, B.; Lynch, J.; Ericson, S.; Freytes, C.O.; LeMaistre, F.; et al. Fluconazole vs Low-Dose Amphotericin B for the Prevention of Fungal Infections in Patients Undergoing Bone Marrow Transplantation: A Study of the North American Marrow Transplant Group. Bone Marrow Transplant. 2000, 25, 853-859. [CrossRef] [PubMed]

9. Kelsey, S.M.; Goldman, J.M.; McCann, S.; Newland, A.C.; Scarffe, J.H.; Oppenheim, B.A.; Mufti, G.J. Liposomal Amphotericin (AmBisome) in the Prophylaxis of Fungal Infections in Neutropenic Patients: A Randomised, Double-Blind, Placebo-Controlled Study. Bone Marrow Transplant. 1999, 23, 163-168. [CrossRef] [PubMed]

10. Prentice, H.G.; Hann, I.M.; Herbrecht, R.; Aoun, M.; Kvaloy, S.; Catovsky, D.; Pinkerton, C.R.; Schey, S.A.; Jacobs, F.; Oakhill, A.; et al. A Randomized Comparison of Liposomal versus Conventional Amphotericin B for the Treatment of Pyrexia of Unknown Origin in Neutropenic Patients. Br. J. Haematol. 1997, 98, 711-718. [CrossRef] [PubMed]

11. El Cheikh, J.; Castagna, L.; Wang, L.; Esterni, B.; Faucher, C.; Furst, S.; Duran, S.; Berger, P.; Ranque, S.; Mohty, M.; et al. Once-Weekly Liposomal Amphotericin B for Prophylaxis of Invasive Fungal Infection after Graft-versus-Host Disease in Allogeneic Hematopoietic Stem Cell Transplantation: A Comparative Retrospective Single-Center Study. Hematol. Oncol. Stem Cell Ther. 2010, 3, 167-173. [CrossRef]

12. Gubbins, P.O.; Amsden, J.R.; McConnell, S.A.; Anaissie, E.J. Pharmacokinetics and Buccal Mucosal Concentrations of a 15 Milligram per Kilogram of Body Weight Total Dose of Liposomal Amphotericin B Administered as a Single Dose $(15 \mathrm{mg} / \mathrm{kg})$, Weekly Dose $(7.5 \mathrm{mg} / \mathrm{kg})$, or Daily Dose $(1 \mathrm{mg} / \mathrm{kg})$ in Peripheral Stem Cell Tran. Antimicrob. Agents Chemother. 2009, 53, 3664-3674. [CrossRef] [PubMed]

13. Ellis, M. New Dosing Strategies for Liposomal Amphotericin B in High-Risk Patients. Clin. Microbiol. Infect. 2008, 14, 55-64. [CrossRef]

14. Cordonnier, C.; Mohty, M.; Faucher, C.; Pautas, C.; Robin, M.; Vey, N.; Monchecourt, F.; Mahi, L.; Ribaud, P. Safety of a Weekly High Dose of Liposomal Amphotericin B for Prophylaxis of Invasive Fungal Infection in Immunocompromised Patients: PROPHYSOME Study. Int. J. Antimicrob. Agents 2008, 31, 135-141. [CrossRef] [PubMed]

15. Walsh, T.J.; Goodman, J.L.; Pappas, P.; Bekersky, I.; Buell, D.N.; Roden, M.; Barrett, J.; Anaissie, E.J. Safety, Tolerance, and Pharmacokinetics of High-Dose Liposomal Amphotericin B (AmBisome) in Patients Infected with Aspergillus Species and Other Filamentous Fungi: Maximum Tolerated Dose Study. Antimicrob. Agents Chemother. 2001, 45, 3487-3496. [CrossRef] [PubMed]

16. Annino, L.; Chierichini, A.; Anaclerico, B.; Finolezzi, E.; Norata, M.; Cortese, S.; Cassett, M.I.; Fallani, S.; Novelli, A.; Girmenia, C. Prospective Phase II Single-Center Study of the Safety of a Single Very High Dose of Liposomal Amphotericin B for Antifungal Prophylaxis in Patients with Acute Myeloid Leukemia. Antimicrob. Agents Chemother. 2013, 57, 2596-2602. [CrossRef] [PubMed]

17. Hand, E.O.; Ramanathan, M.R. Safety and Tolerability of High-Dose Weekly Liposomal Amphotericin B Antifungal Prophylaxis. Pediatr. Infect. Dis. J. 2014, 33, 835-836. [CrossRef] [PubMed]

18. Luu Tran, H.; Mahmoudjafari, Z.; Rockey, M.; Henry, D.; Grauer, D.; Aljitawi, O.; Abhyankar, S.; Ganguly, S.; Lin, T.; McGuirk, J. Tolerability and Outcome of Once Weekly Liposomal Amphotericin B for the Prevention of Invasive Fungal Infections in Hematopoietic Stem Cell Transplant Patients with Graft-versus-Host Disease. J. Oncol. Pharm. Pract. 2016, 22, 228-234. [CrossRef] 
19. Giannella, M.; Ercolani, G.; Cristini, F.; Morelli, M.; Bartoletti, M.; Bertuzzo, V.; Tedeschi, S.; Faenza, S.; Puggioli, C.; Lewis, R.E.; et al. High-Dose Weekly Liposomal Amphotericin B Antifungal Prophylaxis in Patients Undergoing Liver Transplantation: A Prospective Phase II Trial. Transplantation 2015, 99, 848-854. [CrossRef]

20. Morrissey, C.O.; Schwarer, A.P.; Patil, S.; Kalff, A.; Wei, A.; Walker, P.; Avery, S.; Spencer, A.; De Silva, H. Abstract No: P105. A Randomised Study on the Safety of Daily, Intermittent, or Weekly Administration of 1, 3 or $10 \mathrm{mg} / \mathrm{kg} /$ day of AmBisome as Prophylaxis in Acute Leukaemics. Available online: www.aspergillus.org.uk/content/randomised-study-safety-daily-intermittent-or-weeklyadministration-1-3-or-10-mg-kg\%011 (accessed on 20 November 2020).

21. Cornely, O.A.; Leguay, T.; Maertens, J.; Vehreschild, M.J.G.T.; Anagnostopoulos, A.; Castagnola, C.; Verga, L.; Rieger, C.; Kondakci, M.; Härter, G.; et al. Randomized Comparison of Liposomal Amphotericin B versus Placebo to Prevent Invasive Mycoses in Acute Lymphoblastic Leukaemia. J. Antimicrob. Chemother. 2017, 72, 2359-2367. [CrossRef]

22. Uhlenbrock, S.; Zimmermann, M.; Fegeler, W.; Jürgens, H.; Ritter, J. Liposomal Amphotericin B for Prophylaxis of Invasive Fungal Infections in High-Risk Paediatric Patients with Chemotherapy-Related Neutropenia: Interim Analysis of a Prospective Study. Mycoses 2001, 44, 455-463. [CrossRef]

23. Mattiuzzi, G.N.; Estey, E.; Raad, I.; Giles, F.; Cortes, J.; Shen, Y.; Kontoyiannis, D.; Koller, C.; Munsell, M.; Beran, M.; et al. Liposomal Amphotericin B versus the Combination of Fluconazole and Itraconazole as Prophylaxis for Invasive Fungal Infections during Induction: Chemotherapy for Patients with Acute Myelogenous Leukemia and Myelodysplastic Syndrome. Cancer 2003, 97, 450-456. [CrossRef]

24. El-Cheikh, J.; Faucher, C.; Fürst, S.; Duran, S.; Berger, P.; Vey, N.; Stoppa, A.M.; Bouabdallah, R.; Gastaut, J.A.; Viens, P.; et al. High-Dose Weekly Liposomal Amphotericin B Antifungal Prophylaxis Following Reduced-Intensity Conditioning Allogeneic Stem Cell Transplantation. Bone Marrow Transplant. 2007, 39, 301-306. [CrossRef]

25. Penack, O.; Schwartz, S.; Martus, P.; Reinwald, M.; Schmidt-Hieber, M.; Thiel, E.; Blau, I.W. Low-Dose Liposomal Amphotericin B in the Prevention of Invasive Fungal Infections in Patients with Prolonged NeutropeniA: Results from a Randomized, Single-Center Trial. Ann. Oncol. 2006, 17, 1306-1312. [CrossRef] [PubMed]

26. Mattiuzzi, G.; Kantarjian, H.; Ho, J.; Garcia-Manero, G.; Cortes, J. Antifungal Prophylaxis (AFP) for Patients (Pts) with Acute Myelogenous Leukemia (AML) and High-Risk Myelodysplastic Syndrome (HR-MDS) Undergoing Intensive Chemotherapy: An Experience with 730 Pts. Blood 2009, 114, 3102. [CrossRef]

27. Bochennek, K.; Tramsen, L.; Schedler, N.; Becker, M.; Klingebiel, T.; Groll, A.H.; Lehrnbecher, T. Liposomal Amphotericin B Twice Weekly as Antifungal Prophylaxis in Paediatric Haematological Malignancy Patients. Clin. Microbiol. Infect. 2011, 17, 1868-1874. [CrossRef] [PubMed]

28. Chakrabartty, J.; Pavlu, J.; Neave, E.; Eguialde, M.; O'Shea, D.; Giles, C.; Goldman, J.; Kirschke, S.; Kanfer, E.; Kishore, B.; et al. Safety and Efficacy of Two Regimens of High-Dose Liposomal Amphotericin B for Prophylaxis of Invasive Fungal Infection in Allogeneic Haematopoietic Stem Cell Transplantation: A Comparative Retrospective Single-Centre Study. In Proceedings of the 38th Annual Meeting of the European Group for Blood and Marrow Transplantation (EBMT), Geneva, Switzerland, 1-4 April 2012; p. S192.

29. U.S. Department of Health and Human Services, National Institutes of Health, N.C.I. Common Terminology Criteria for Adverse Events (CTCAE). Available online: https://ctep.cancer.gov/protocolDevelopment/ electronic_applications/docs/CTCAE_v5_Quick_Reference_8.5x11.pdf (accessed on 20 November 2020).

30. De Pauw, B.; Walsh, T.J.; Donnelly, P.; Stevens, D.A.; Edwards, J.E.; Calandra, T.; Pappas, P.G.; Maertens, J.; Lortholary, O.; Kauffman, C.A.; et al. Revised Definitions of Invasive Fungal Disease from the European Organization for Research and Treatment of Cancer/Invasive Fungal Infections Cooperative Group and the National Institute of Allergy and Infectious Diseases Mycoses Study Group (EORTC/MSG) C. Clin. Infect. Dis. 2008, 46, 1813-1821. [CrossRef] [PubMed]

31. Cornely, O.A.; Hoenigl, M.; Lass-Flörl, C.; Chen, S.C.A.; Kontoyiannis, D.P.; Morrissey, C.O.; Thompson, G.R. Defining Breakthrough Invasive Fungal Infection-Position Paper of the Mycoses Study Group Education and Research Consortium and the European Confederation of Medical Mycology. Mycoses 2019, 62, 716-729. [CrossRef] [PubMed]

32. Health Research Authority. Do I Need NHS REC Review? Available online: http://www.hra-decisiontools. org.uk/ethics/ (accessed on 20 November 2020). 
33. Billings Iv, F.T.; Shaw, A.D. Clinical Trial Endpoints in Acute Kidney Injury. Nephron. Clin. Pract. 2014, 127, 89-93. [CrossRef] [PubMed]

34. Chamilos, G.; Lionakis, M.S.; Kontoyiannis, D.P. Call for Action: Invasive Fungal Infections Associated with Ibrutinib and Other Small Molecule Kinase Inhibitors Targeting Immune Signaling Pathways. Clin. Infect. Dis. 2018, 66, 140-148. [CrossRef]

35. Teh, B.W.; Chui, W.; Handunnetti, S.; Tam, C.; Worth, L.J.; Thursky, K.A.; Slavin, M.A. High Rates of Proven Invasive Fungal Disease with the Use of Ibrutinib Monotherapy for Relapsed or Refractory Chronic Lymphocytic Leukemia. Leuk. Lymphoma 2019, 60, 1572-1575. [CrossRef]

36. Amplyx (C): Our Pipeline. Available online: https://amplyx.com/our-pipeline/ (accessed on 20 November 2020).

37. Gebremariam, T.; Alkhazraji, S.; Gu, Y.; Singh, S.; Alqarihi, A.; Shaw, K.J.; Ibrahim, A.S. Galactomannan Is a Biomarker of Fosmanogepix (APX001) Efficacy in Treating Experimental Invasive Pulmonary Aspergillosis. Antimicrob. Agents Chemother. 2020, 64, 17-20. [CrossRef]

38. F2G. F2G Pipeline. Available online: https://www.f2g.com (accessed on 20 November 2020).

39. Seyedmousavi, S.; Chang, Y.C.; Law, D.; Birch, M.; Rex, J.H.; Kwon-Chung, K.J. Efficacy of Olorofim (F901318) against Aspergillus fumigatus, A. Nidulans, and A. Tanneri in Murine Models of Profound Neutropenia and Chronic Granulomatous Disease. Antimicrob. Agents Chemother. 2019, 63, 1-11. [CrossRef]

40. Davis, M.R.; Donnelley, M.A.; Thompson, G.R. Ibrexafungerp: A Novel Oral Glucan Synthase Inhibitor. Med. Mycol. 2020, 58, 579-592. [CrossRef] [PubMed]

41. Duckwall, M.J.; Gales, M.A.; Gales, B.J. Inhaled Amphotericin B as Aspergillosis Prophylaxis in Hematologic Disease: An Update. Microbiol. Insights 2019, 12, 117863611986993. [CrossRef] [PubMed]

42. Smith, P.J.; Olson, J.A.; Constable, D.; Schwartz, J.; Proffitt, R.T.; Adler-Moore, J.P. Effects of Dosing Regimen on Accumulation, Retention and Prophylactic Efficacy of Liposomal Amphotericin B. J. Antimicrob. Chemother. 2007, 59, 941-951. [CrossRef] [PubMed]

43. Garcia, A.; Adler-Moore, J.P.; Proffitt, R.T. Single-dose AmBisome (Liposomal Amphotericin B) as Prophylaxis for Murine Systemic Candidiasis and Histoplasmosis. Antimicrob. Agents Chemother. 2000, 44, 2327-2332. [CrossRef] [PubMed]

44. Bicanic, T.; Bottomley, C.; Loyse, A.; Brouwer, A.E.; Muzoora, C.; Taseera, K.; Jackson, A.; Phulusa, J.; Hosseinipour, M.C.; Van Der Horst, C.; et al. Toxicity of Amphotericin B Deoxycholate-Based Induction Therapy in Patients with HIV-Associated Cryptococcal Meningitis. Antimicrob. Agents Chemother. 2015, 59, 7224-7231. [CrossRef]

45. Youngs, J.; Bicanic, T. Aspergillosis in Patients with Severe Influenza or Coronavirus (AspiFlu). Available online: http://www.isrctn.com/ISRCTN51287266 (accessed on 20 November 2020).

Publisher's Note: MDPI stays neutral with regard to jurisdictional claims in published maps and institutional affiliations.

(C) 2020 by the authors. Licensee MDPI, Basel, Switzerland. This article is an open access article distributed under the terms and conditions of the Creative Commons Attribution (CC BY) license (http://creativecommons.org/licenses/by/4.0/). 